\title{
Estudio de la eficacia de sellado en el periodo interdialítico largo para catéteres de hemodiálisis disfuncionantes
}

\author{
Patricia Arribas Cobo, Pilar Rodríguez Gayán, José Alfredo Sáenz Santolaya, Mª Trinidad Quesada Armenteros, \\ Patricia Molina Mejías, Cristina Quero Lope
}

Hospital Universitario Infanta Leonor. Madrid. España

\begin{abstract}
Resumen
El sellado de los catéteres venosos tunelizados con distintas soluciones se ha utilizado tanto en la prevención como en el tratamiento de complicaciones, sin embargo, no existe la solución ideal. El objetivo del estudio es comparar las complicaciones de de los catéteres disfuncionantes con diferentes pautas de sellado de fin de semana, Urokinasa 10.000 UI vs TauroLock ${ }^{\mathrm{TM}}-\mathrm{U} 25.000$, así como eficacia dialítica de los mismos. Se trata de un estudio observacional longitudinal de cohorte en el que se analizan los casos de infección, trombosis y funcionalidad del catéter tunelizado. Se incluyen sólo los pacientes con catéter disfuncionante que tenían previamente prescrita una pauta de sellado con urokinasa en el periodo interdialítico largo. El periodo de estudio fue de cuatro meses; dos con sellado con 10.000 UI de Urokinasa y dos con sellado de TauroLock ${ }^{\mathrm{TM}}$-U25.000. Las medias de Urokinasa vs Taurolidina: flujo sanguíneo, PV, recirculación, KT (53.3/55L), manipulación y dosis extra de urokinasa fueron mejor en el periodo de Taurolock, aunque no se encontraron diferencias estadísticamente significativas. La media de PA es cercana a la significación ( $p=0.067)$ mejor también en el periodo de Taurolock. Hubo una diferencia en la media de KT favorable al periodo de Taurolock que no tiene significación estadística y es exclusivo de los pacientes que no necesitaron urokinasa extra. La principal limitación de nuestro estudio es el tamaño muestral que puede ser el responsable en parte, de la ausencia de significación estadística. Son necesarios estudios con mayor número de pacientes.
\end{abstract}

Correspondencia:

Patricia Arribas Cobo

C/ Florencia 8, $1{ }^{\circ}$ A. 28030 Madrid. España

E-mail:patriarribas@gmail.com
PALABRAS CLAVE

- CATÉTER

- SELLADO

- HEPARINA

- TAUROLIDINA

Study of sealing efficiency in the long interdialytic period for dysfunctioning hemodialysis catheters

\section{Abstract}

The sealing of the tunneled venous catheters with different solutions has been used both in prevention and in the treatment of complications, however, there is no ideal solution. The aim of the study is to compare the complications of dysfunctional catheters with different weekend sealing patterns, Urokinase 10,000 IU vs TauroLock ${ }^{\mathrm{TM}}-\mathrm{U} 25,000$, as well as dialytic efficacy of both substances. A longitudinal observational cohort study in which the cases of infection, thrombosis and functionality of the tunneled catheter were analyzed. Only patients with a dysfunctioning catheter who had previously prescribed a sealing pattern with urokinase in the long interdialytic period were included. The study period was four months; two months sealed with 10,000 IU of Urokinase and the other ones, sealed with TauroLock TM -U25,000. Urokinase vs Taurolidine averages: blood flow, venous pressure, recirculation, KT (53.3 / 55L), manipulation and extra doses of urokinase were better in the Taurolock period, although no statistically significant differences were found. The mean arterial pressure is close to the significance ( $p$ $=0.067$ ), also better in the Taurolock period. There was a difference in the KT mean, favorable to the Taurolock period, which has no statistical significance and is exclusive to patients who did not require extra urokinase. The main limitation of our study is the 
sample size that may be partly responsible for the absence of statistical significance. Studies with more patients are necessary.

\section{KEYWORDS}

- CATHETER

- SEALED

- HEPARIN

- TAUROLIDINE

\section{Introducción}

En los últimos años se ha incrementado el porcentaje de pacientes portadores de catéter venoso tunelizado (CVT) como acceso vascular para hemodiálisis. Sus principales complicaciones son la disfunción y las infecciones $y$, además el uso de catéteres tunelizados es un factor independientemente asociado con la mortalidad de los pacientes ${ }^{1}$.

El sellado de los CVT con distintas soluciones se ha utilizado tanto en la prevención como en el tratamiento de estas complicaciones, sin embargo, no existe la solución ideal.

La trombosis y la disfunción suponen un aumento en las manipulaciones que aumentan el riesgo de infección, el uso de agentes trombolíticos, así como empeoramiento de su funcionalidad, lo que implica mayor mortalidad y una disminución de la eficacia dialítica.

La solución ideal para realizar el sellado del CVT debería tener las siguientes características: ser biocompatible, no tóxico, seguro, gran actividad anticoagulante, antimicrobiano, capacidad para prevenir 0 destruir el biofilm, económico de fácil manipulación y manejo para el personal de enfermería y sin efectos secundarios.

No existe por esto el producto de sellado ideal y no hay consenso sobre el tipo ni sobre sus dosis, aunque sí recomendaciones desde las distintas guías de actuación.

En las Guías de Accesos Vasculares recientemente publicadas se sugiere el sellado del CVT durante los periodos entre sesiones se realice con heparina, con citrato o con activador tisular del plasminógeno al- ternando con heparina. En el caso de disfunción por trombosis se recomienda el tratamiento con infusión intraluminal de fibrinolíticos².

Por otro lado hay que definir cuando consideramos a un CVT disfuncionante. La recomendación es considerarlo así cuando no se consigue obtener o mantener un flujo de sangre extracorpóreo adecuado $(\mathrm{Qb}>300$ $\mathrm{mil} / \mathrm{min}$ ) para realizar la hemodiálisis.

Para intentar mejorar los porcentajes de permeabilidad tras la infusión de UK, Twardowski propone en 1998 la utilización de UK en altas dosis y en infusión continua, $250.000 \mathrm{U}$ durante 3 horas $^{3}$. Sin embargo, no hay recomendación sobre el sellado de CVT en aquellos que se muestran disfuncionantes con cierta frecuencia, es decir, que precisan tratamiento con fibrinolíticos al menos de forma mensual.

En nuestra unidad hay un elevado porcentaje de CVT $(29.48 \%)$ de los cuales un $30.43 \%$ detectamos que son disfuncionantes. En éstos pacientes utilizamos seIlados con Urokinasa 10.000 UI en lugar de heparina $1 \%$ durante el periodo de fin de semana, al margen del tratamiento fibrinolítico con urokinasa en el momento puntual de presentar disfunción.

La urokinasa no está exenta de efectos secundarios y contraindicaciones $y$, además, presenta una actividad anticoagulante solo de 24 horas tras su administración ${ }^{4}$, por lo que nos planteamos compararlo con el uso de otro producto compuesto por taurolidina con urokinasa (Taurolock ${ }^{\mathrm{TM}}-\mathrm{U} 25.000$ ), que además de ser un compuesto antimicrobiano, contiene citrato al $4 \%$ como anticoagulante. A esta concentración elimina el calcio de manera segura y eficaz de la cascada de la coagulación y que contiene además $5.000 \mathrm{UI} / \mathrm{ml}$ de uroquinasa ${ }^{5}$.

El objetivo del estudio es comparar las complicaciones de los catéteres disfuncionantes con diferentes pautas de sellado de fin de semana, Urokinasa 10.000 UI vs TauroLock ${ }^{\mathrm{TM}}$-U25.000, así como eficacia dialítica de los mismos.

\section{Material y Método}

Se trata de un estudio observacional longitudinal de cohorte en el que se analizan los casos de infección, trombosis y funcionalidad del CVT. 
Se incluyen sólo los pacientes con catéter disfuncionante que tenían previamente prescrita una pauta de sellado con urokinasa en el periodo interdialítico largo.

El periodo de estudio fue de cuatro meses; durante los dos primeros meses se realiza un sellado con sellado con 10.000 UI de Urokinasa y los dos meses siguientes se realiza el sellado con sellado de TauroLock $^{\mathrm{TM}}$-U25.000.

Se estudian distintas variables clínicas como edad, etiología de la enfermedad renal, índice de comorbilidad de Charlson y sexo, así como variables de funcionamiento del CVT como flujo de sangre, presión arterial, presión venosa del circuito, recirculación, número de manipulaciones, número de dosis extra de urokinasa, número de bacteriemias y la eficacia dialítica (KT).

La recirculación es la medida por el monitor de Fresenius ST5008 en el que se dializaban 6 de los 7 pacientes, por lo que los valores son los registrados solo de 6 pacientes del estudio.

El número de manipulaciones se estipula por el número de veces que se precisa conectar el catéter con las ramas invertidas o las veces que se precisa parar la diálisis para realizar lavados o cambios de conexión de las ramas.

La dosis extra de urokinasa la definimos por el número de veces que se precisa el uso de la misma de forma puntual por disfunción sin contar con el sellado del fin de semana.

Se creó una hoja excell de recogida de datos para las variables de funcionamiento y se recogieron los datos de las variables clínicas de las historias clínicas de los pacientes. Se utilizó la t-Student para relacionar las variables cuantitativas. Se utilizó el programa SPSS.15 para Windows para el procesamiento de los datos.

\section{Resultados}

La muestra la constituyeron 7 pacientes, $4(57,1 \%)$ hombres y $3(42,9 \%)$ mujeres, con una edad media de 76,1 [63-94] años.
Se estudiaron un total de 364 sesiones de diálisis (26 sesiones por paciente con cada tipo de sellado) durante los cuatro meses de estudio.

La media del tiempo en diálisis era de 80.2 meses [10250]. La media del índice de comorbilidad de Charlson fue de 6.86 [3-12].

La causa de enfermedad renal era variable, siendo la principal la diabética $3(42,9 \%)$, pielonefritis $1(14.3 \%)$, poliquistosis $1(14.3 \%)$, nefrotoxicidad $1(14.3 \%)$, nefroangioesclerosis 1 ( $14.3 \%)$.

Las medias de Urokinasa vs Taurolidina de las variables funcionales se presentan en la tabla 1.

\begin{tabular}{ccc}
$\begin{array}{c}\text { PERIODO } \\
\text { UROKINASA }\end{array}$ & $\begin{array}{c}\text { PERIODO } \\
\text { TAUROLIDINA UK }\end{array}$ & $\begin{array}{c}\text { SIGNIFICACIÓN } \\
\text { ESTADISTICA }\end{array}$ \\
\hline $340 \mathrm{~m} / \mathrm{min}$ & $344 \mathrm{ml} / \mathrm{min}$ & $\mathrm{n}=0.679$ \\
$-204 \mathrm{mmHg}$ & $-198,4 \mathrm{mmHg}$ & $\mathrm{n}=0.067$ \\
$157 \mathrm{mmHg}$ & $150 \mathrm{mmHg}$ & $\mathrm{n}=0.335$ \\
$14,8 \%$ & $14,5 \%$ & $\mathrm{n}=0.781$ \\
2,5 veces & 1,5 & $\mathrm{n}=0.193$ \\
1,21 & 1,14 & $\mathrm{n}=0.679$ \\
53,3 & 55 & $\mathrm{n}=0.887$ \\
\hline
\end{tabular}

\section{Discusión y conclusiones}

Las medias de los valores de flujo sanguíneo $(340 / 344 \mathrm{ml} / \mathrm{min}), \mathrm{PV}(157 / 150 \mathrm{mmHg})$, recirculación $(14.8 / 14.5 \%)$, KT $(53.3 / 55 \mathrm{~L})$, manipulación (2.5/1.5) y dosis extra de urokinasa (1.21/1.14) fueron mejor en el periodo de Taurolock, aunque no se encontraron diferencias estadísticamente significativas. La media de PA $(-204 /-198.4 \mathrm{~mm} \mathrm{Hg})$ es cercana a la significación ( $p=0.067)$ mejor también en el periodo de Taurolock.

Hubo una diferencia en la media de KT de 1.7 litros favorable al periodo de Taurolock que no tiene significación estadística y es exclusivo de los pacientes que no necesitaron urokinasa extra.

No hubo ninguna bacteriemia en ninguno de los periodos de estudio, si bien esta tasa en nuestra unidad es muy baja $(0,25 / 1000$ días de catéter).

En nuestro estudio encontramos que hay una cierta mejora en los parámetros de funcionalidad y eficacia 
dialítica de los CTV sellados con Taurolidina aunque sin significación estadística.

La principal limitación de nuestro estudio es el pequeño tamaño muestral que puede ser el responsable en parte, de la ausencia de significación estadística. Serán necesarios estudios con mayor número de pacientes para poder hallar resultados más concluyentes.

Recibido: 15 enero 2017

Revisado: 21 enero 2017

Modificado: 10 febrero 2017

Aceptado: 13 febrero 2017

\section{Bibliografía}

1. Gruss y cols. Repercusiones del uso de catéteres en hemodiálisis. Nefrología 29, 2: 13-129; 2009.

2. Guía Española de AccesoVascular para Hemodiálisis. GEMAV. [acceso el 7 de julio de 2016.] Disponible en: http://www.senefro.org/modules. php? name $=$ webstructure \&idwebstructure $=36$

3. Twardowski ZJ. The clotted central vein catheter for hemodialysis. Nephrol Dial Transplant. 1998;13:2203-2206.

4. Ficha técnica urokinasa 2016. [acceso el 7 de julio de 2016.] Disponible en: https://www.aemps.gob. es/cima/pdfs/es/ft/55422/55422_ft.pdf

5. Ficha técnica TauroLock ${ }^{\mathrm{TM}}$-U25.000. [acceso el 7 de julio de 2016.] Disponible en: http://www. taurolock.com/es 